\title{
Insight into the Quality of Life of Patients with Ankylosing Spondylitis: Real-World Data from a US-Based Life Impact Survey
}

\author{
James T. Rosenbaum • Lisa Pisenti - Yujin Park • Richard A. Howard
}

Received: January 7, 2019 / Published online: May 20, 2019

(C) The Author(s) 2019

\begin{abstract}
Introduction: We aim to assess the real-world, US patient-reported impact of ankylosing spondylitis (AS) on quality of life (QOL) across physical, discomfort, social, and emotional domains.

Methods: Demographic and QOL data were collected from a random sample of patients associated with the Spondylitis Association of America (SAA) from July to December 2017.

Enhanced Digital Features To view enhanced digital features for this article go to https://doi.org/10.6084/ m9.figshare.8047292.
\end{abstract}

Electronic supplementary material The online version of this article (https://doi.org/10.1007/s40744019-0160-8) contains supplementary material, which is available to authorized users.

J. T. Rosenbaum ( $₫)$

Oregon Health \& Science University, Portland, OR, USA

e-mail: rosenbaj@ohsu.edu

J. T. Rosenbaum

Legacy Devers Eye Institute, Portland, OR, USA

L. Pisenti

UCB, Inc., Smyrna, GA, USA

Y. Park

Novartis Pharmaceuticals Corporation, East

Hanover, NJ, USA

R. A. Howard

Spondylitis Association of America, Van Nuys, CA, USA
QOL measures were based on the Evaluation of Ankylosing Spondylitis Quality of Life (EASiQoL) questionnaire. The survey evaluated AS impact on the physical domain on the day of survey participation and impact on the discomfort, social, and emotional domains within the week before participation. A 3:1 (male to female) weighting was performed to reflect the reported prevalence of AS in US adults.

Results: Of 820 respondents who completed the survey, 716 self-reported receiving an AS diagnosis from their doctor and were included in this analysis (mean age, 55.5 years; $46.9 \%$ male). The mean total EASi-QoL score was 28.9 (weighted); overall, 33.7\%, 31.7\%, and $34.7 \%$ of respondents, respectively, reported a low (EASiQoL score $0-17)$, a medium (18-35), and a high $(\geq 36)$ impact of AS on QOL. The physical domain was most impacted; $41.9 \%$ of respondents had an EASi-QoL score $\geq 10$ (weighted). Women were significantly more likely than men to report a high impact of AS on all QOL domains. Biologic users reported an impact on QOL comparable with the impact on QOL of nonsteroidal anti-inflammatory drug use. AS also impacted lifestyle characteristics, including career choice and sports participation.

Conclusion: AS negatively impacted all QOL domains analyzed. The incorporation of subjective measures of disease into disease evaluation should be considered.

Funding: Novartis Pharmaceuticals Corporation and UCB, Inc. 
Plain Language Summary: Plain language summary available for this article.

Keywords: Ankylosing

spondylitis; Epidemiological monitoring; Patient-reported outcomes; Quality of life; Qualitative research

\section{PLAIN LANGUAGE SUMMARY}

Ankylosing spondylitis (AS) is a devastating disease with a negative impact on patients' quality of life (QOL). In our study, 716 patients with AS across the United States reported the consequences of their disease on their QOL in a survey. Patients answered questions on how AS affected them in 4 ways: physical (e.g., lifting a child or heavy objects), discomfort (e.g., feeling tired or lacking energy), emotional (e.g., worrying about the future), and social (e.g., feeling downhearted or low). In the survey, patients were asked how AS impacted them physically on the day of survey participation, and how AS caused them discomfort and affected them emotionally and socially within the past week before survey participation.

Overall, similar numbers of patients (approximately 3 out of 10 ) reported a low, a medium, and a high impact of AS on their QOL, respectively. Patients reported the most impact physically. More women with AS reported a negative impact of the disease on all aspects (physical, discomfort, emotional, and social) compared with men. AS also affected patients' work and lifestyle; for instance, approximately 3 out of 10 patients reported that their disease negatively affected their participation in sports all the time. Our study provides insight into the patient-reported impact of AS on their QOL.

\section{INTRODUCTION}

Spondyloarthritis (SpA) is a chronic inflammatory rheumatic disease that may be predominantly axial or predominantly peripheral; axial SpA encompasses ankylosing spondylitis (AS), which exhibits radiological signs of sacroiliitis [1]. Current estimates indicate that AS affects $0.20-0.55 \%$ of the general population in the
United States [2]. Patients with AS often experience impairment or loss of physical function [3] and are usually at higher risk of developing many comorbidities, including cardiovascular disease, osteoporosis, and uveitis, compared with the general population [4-8]. The goals of treatment in patients with AS are to reduce and/ or control inflammation, pain, stiffness, and fatigue; maintain spinal flexibility and normal posture; reduce functional limitations; maintain social relationships and work ability; and decrease complications of disease $[9,10]$. Nonsteroidal anti-inflammatory drugs (NSAIDs) are the recommended first-line treatment of AS $[9,10]$. However, in inadequate responders or patients experiencing the side effects of NSAIDs, biologics have emerged as novel anti-inflammatory therapies [11].

Early indicators of AS may not be discernible; hence, patients often have chronic pain due to an "invisible" disease [12]. Qualitative studies revealed the perception of "having an unfamiliar body" [13]; while physical pain interfered with daily living, the psychosocial outcomes of pain-such as distress, loneliness, identity loss, and low quality of life (QOL)—often interfered with treatment $[14,15]$. The delay between symptom onset and diagnosis of disease has been reported to be approximately 13 years in the United States [16] and the presence of a lag has been attributed to frequent presentation of back pain and the lack of clinical symptoms or signs unique to AS $[17,18]$. Impairment of QOL, increased mortality, and substantial healthcare costs impose a high burden on patients, their caregivers, and society [19].

A survey of 592 patients with rheumatic diseases, including AS, indicated that up to 50\% of patients were seldom or never queried about the impact of their disease on their QOL, and that normalization of QOL and symptom relief were the most important treatment attributes for these patients [20]. A patient's self-reported QOL is typically assessed by more generic instruments, such as the Medical Outcomes Study 36-Item Short Form [21], to provide an overall QOL summary, or by disease-specific instruments, such as the Ankylosing Spondylitis Quality of Life [22] and the Evaluation of Ankylosing Spondylitis Quality of Life (EASi- 
QoL) [23] questionnaires. Several studies have used both generic and disease-specific instruments to evaluate the QOL of patients with AS and identified several predictors of QOL, including demographic variables, disease parameters, psychosocial factors, and advances in AS treatment and management [24-27]. As randomized clinical trials often comprise select groups of patients who are rigorously monitored, limited information is available about real-world patient QOL.

The Spondylitis Association of America (SAA), founded in 1983, is a national nonprofit organization dedicated to patient care, research, and prevention of AS and related diseases. The SAA first conducted the AS Life Impact Survey (LIS) in 2002, before the era of biologic treatment for AS, to uncover the impact of AS on patients' lives; this study revealed delays in AS diagnosis as well as functional impairment and work disability among patients with AS [28]. Later, in 2011, another LIS was conducted to reassess patient QOL [29]. The data derived from these studies represent evidence obtained from clinical practice settings among a heterogenous patient population. In this 2017 LIS, we sought to update our understanding of the impact of AS by evaluating the patient-reported impact of AS on QOL in the physical, discomfort, social, and emotional domains among US patients with AS in a real-world setting.

\section{METHODS}

\section{SAA LIS Study Design and Data Source}

A cross-sectional survey was conducted among patients with a diagnosis of SpA in the SAA database, comprising sources such as the SAA website, physician referrals, seminar attendees, webinars, in-person support group meetings, word of mouth, health fairs, outdoor advertising, press releases, and social media outreach efforts. For the 2017 LIS, we initially aimed for a total of 800 final enrollees (700 online and 100 by phone). A total of 820 interviews were conducted with SAA contacts (20 additional respondents were enrolled as they completed the survey in the time it took to shut the online survey down) between July 7, 2017 and December 31, 2017; a flow diagram summarizing the survey participant recruitment and follow-up is provided in Fig. 1. To provide additional context for how SpA has impacted QOL over time, findings from the 2011 LIS were also analyzed and are presented in the supplement. For the 2011 LIS, a total of 865 interviews were conducted; 757 were conducted online and 108 were conducted by phone in July 2011. Respondents were randomly selected by assigning a number to each person initiating contact with the SAA and then choosing random numbers for participation. Each person in the randomly selected group was screened for the presence of a self-reported SpA diagnosis (including AS, undifferentiated spondyloarthropathy, psoriatic arthritis, axial SpA, enteropathic arthritis associated with inflammatory bowel disease, and juvenile spondyloarthropathy). This study was designed, conducted, and reported in accordance with the Declaration of Helsinki and the Belmont Report.

\section{Data Variables}

Each respondent answered questions regarding baseline demographics, including age, sex, race, current working status, insurance type, and geographic region. All respondents self-reported their physician-made SpA diagnosis, which included AS, uveitis/iritis, undifferentiated SpA, psoriatic arthritis, axial SpA, enteropathic arthritis associated with inflammatory bowel disease, reactive arthritis, and juvenile SpA. Respondents selected any of the aforementioned SpA conditions that applied to them; it is possible that respondents may have had overlapping SpA conditions. The respondents also reported the duration of their disease, the time since diagnosis, and a description of the doctor care that they were receiving for their SpA (i.e., physician specialty, number of doctors seen, frequency of visits to a doctor). The respondents also reported specific locations of joint involvement as well as any existing comorbidities or other associated conditions, such as high blood pressure, high cholesterol, depression, 


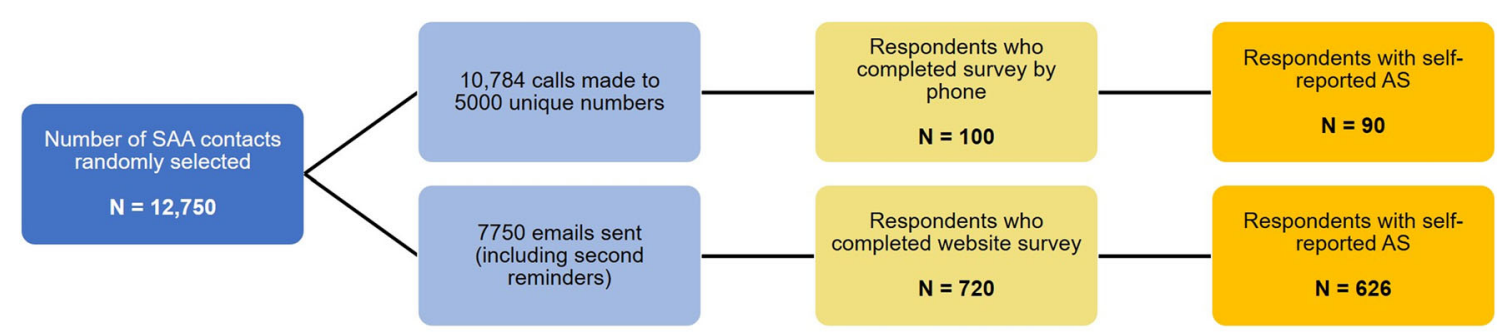

Fig. 1 A study flow diagram illustrating the number of SAA contacts randomly selected for participation in the study, the number of phone calls made and emails sent,

fibromyalgia, Crohn disease/ulcerative colitis, acid reflux, eye inflammation, irritable bowel syndrome, migraine, and balance issues. The respondents specified the treatment agents that they were receiving at the time of survey participation, including analgesics, biologics, cannabis, NSAIDs, opioids, slow-acting antirheumatic drugs, and steroids.

The QOL of respondents with AS was assessed based on the EASi-QoL questionnaire [23]. The EASi-QoL consists of 20 questions designed to evaluate the impact of AS on four QOL domains (scale 0-80; a higher score indicates a severer impact): physical function (6 questions; maximum score $=24$ ), disease activity or discomfort (4 questions; maximum score $=16$ ), emotional well-being (5 questions; maximum score $=20$ ), and social participation (5 questions; maximum score $=20$ ). Each question is scored $0-4 \quad(0=$ not limited at all, $1=$ a little limited, $2=$ moderately limited, $3=$ very limited, and $4=$ totally limited/unable to do). A low impact of disease on QOL corresponds to a total EASi-QoL score of 0-17, a medium impact to a score of $18-35$, and a high impact to a score of $\geq 36$. The top two levels of impairment, i.e., "very limited" and "totally limited/unable to do," were considered a high impact of disease for each individual question. Participants were queried about the impact of AS on their QOL within the day of survey participation with regard to the physical domain and within the week prior to participation with regard to the discomfort, emotional, and social domains. Although AS affects men more commonly than women, $53.1 \%$ of the survey respondents were women [30]. Accordingly, a 3:1 (male to female) and the number of completed survey responses. $A S$ ankylosing spondylitis; $S A A$ Spondylitis Association of America

weighting was performed for select outcomes to reflect the reported prevalence of AS in US adults [30].

\section{Data Analysis}

Categorical variables were presented as the count and percentage of respondents per category, and continuous variables were summarized with means. Outcomes from the 2017 LIS were presented for overall respondents with AS (weighted) and for respondents with AS stratified by sex (unweighted). Outcomes from the 2011 LIS were presented for overall respondents with AS (weighted) and for respondents with AS stratified by sex (unweighted). The chi-square test was used to test for differences in categorical variables between men and women; $P<0.05$ was considered statistically significant.

\section{Compliance with Ethics Guidelines}

IRB approval was not obtained when this study was designed in 2011. The IRB of Oregon Health \& Science University has since confirmed that the study design was compliant with the Declaration of Helsinki and the Belmont Report.

\section{RESULTS}

Of the 820 participants who completed the 2017 LIS, 716 (87.3\%) self-reported receiving a diagnosis of AS from their doctor and were included in this study. Baseline demographics and disease characteristics are summarized in 
Table 1 Baseline demographics and disease characteristics of respondents with AS in the 2017 Life Impact Survey

\begin{tabular}{|c|c|}
\hline Characteristic & $\begin{array}{l}\text { Respondents with } \\
\text { AS, } N=716\end{array}$ \\
\hline Age, mean, years & 55.5 \\
\hline Age category, $n(\%)$ & $N=716$ \\
\hline$<35$ years & $63(8.8)$ \\
\hline 35 to $<55$ years & $239(33.4)$ \\
\hline$\geq 55$ years & $412(57.5)$ \\
\hline Unsure/missing & $2(0.3)$ \\
\hline Male, $n(\%)$ & $336(46.9)$ \\
\hline Race, $n(\%)$ & $N=716$ \\
\hline White & $641(89.5)$ \\
\hline African American & $11(1.5)$ \\
\hline Asian American & $8(1.1)$ \\
\hline Other/unsure & $56(7.8)$ \\
\hline Work status, $n(\%)$ & $N=716$ \\
\hline Full time & $278(38.8)$ \\
\hline Part time & $97(13.5)$ \\
\hline Retired & $227(31.7)$ \\
\hline Not working & $102(14.2)$ \\
\hline Other & $67(9.4)$ \\
\hline Insurance type, $n(\%)$ & $N=716$ \\
\hline $\mathrm{PPO}$ & $294(41.1)$ \\
\hline Medicare & $241(33.7)$ \\
\hline Third party & $113(15.8)$ \\
\hline $\mathrm{HMO}$ & $97(13.5)$ \\
\hline Other & $196(27.4)$ \\
\hline None & $9(1.3)$ \\
\hline US region, $n(\%)$ & $N=716$ \\
\hline Northeast & $145(20.3)$ \\
\hline Southeast & $155(21.6)$ \\
\hline Midwest & $131(18.3)$ \\
\hline West & $195(27.2)$ \\
\hline Southwest & $81(11.3)$ \\
\hline
\end{tabular}

Table 1 continued

\begin{tabular}{|c|c|}
\hline Characteristic & $\begin{array}{l}\text { Respondents with } \\
\text { AS, } N=716\end{array}$ \\
\hline Other (out of country) & $9(1.3)$ \\
\hline Locations of pain, $n(\%)$ & $N=716$ \\
\hline Lumbar spine & $622(86.9)$ \\
\hline Neck & $602(84.1)$ \\
\hline Hip joint & $574(80.2)$ \\
\hline Waist or sacrum or pelvis & $505(70.5)$ \\
\hline Shoulders & $496(69.3)$ \\
\hline Comorbidities, $n(\%)$ & $N=705$ \\
\hline High blood pressure & $253(35.9)$ \\
\hline High cholesterol & $188(26.7)$ \\
\hline Depression & $170(24.1)$ \\
\hline Fibromyalgia & $98(13.9)$ \\
\hline Associated diseases, $n$ (\%) & $N=716$ \\
\hline Uveitis & $217(30.3)$ \\
\hline Crohn disease or ulcerative colitis & $110(15.6)$ \\
\hline Other conditions, $n(\%)$ & $N=716$ \\
\hline Acid reflux & $364(50.8)$ \\
\hline Eye inflammation & $332(46.4)$ \\
\hline Irritable bowel syndrome & $239(33.4)$ \\
\hline Migraine & $237(33.1)$ \\
\hline Balance issues & $234(32.7)$ \\
\hline $\begin{array}{l}\text { Mean time since the development } \\
\text { of symptoms associated with AS, } \\
\text { years }\end{array}$ & 26.6 \\
\hline $\begin{array}{l}\text { Time since the development of } \\
\text { symptoms associated with AS, } \\
n(\%)\end{array}$ & $N=716$ \\
\hline$\leq 5$ years ago & $69(9.6)$ \\
\hline 6 to $<20$ years ago & $216(30.2)$ \\
\hline$\geq 20$ years ago & $431(60.2)$ \\
\hline $\begin{array}{l}\text { Mean time since diagnosis of AS, } \\
\text { years }\end{array}$ & 18.4 \\
\hline
\end{tabular}


Table 1 continued

\begin{tabular}{ll}
\hline Characteristic & $\begin{array}{l}\text { Respondents with } \\
\text { AS, } N=716\end{array}$ \\
\hline Time since diagnosis of AS, $n(\%)$ & $N=716$ \\
$\leq 5$ years ago & $162(22.6)$ \\
6 to $<20$ years ago & $272(38.0)$ \\
$\geq 20$ years ago & $282(39.4)$ \\
\hline
\end{tabular}

$A S$ ankylosing spondylitis, HMO health maintenance organization, $P P O$ preferred provider organization

Table 1. The mean age of survey participants was 55.5 years; $46.9 \%$ were male and $89.5 \%$ were white. Approximately $40 \%$ of participants each worked full time and had preferred provider organization insurance plans. Participants were distributed across all regions of the United States (Northeast, 20.3\%; Southeast, 21.6\%; Midwest, 18.3\%; West, 27.2\%; Southwest, $11.3 \%$; other, $1.3 \%$ ). The most common locations of pain reported were the lumbar spine (86.9\%), neck (84.1\%), and hip joint (80.2\%), and the most frequently reported comorbidities were high blood pressure $(35.9 \%)$, high cholesterol $(26.7 \%)$, and depression (24.1\%). Significant sex differences were also noted with several comorbid conditions-more men than women reported high blood pressure $(42.7 \%$ vs $28.5 \%$; $P<0.0001)$ and heart disease $(14.0 \%$ vs $4.0 \%$; $P<0.0001)$, while more women than men reported fibromyalgia $(22.1 \%$ vs $4.2 \%$; $P<0.0001)$ and depression $(27.9 \%$ vs $17.7 \%$; $P=0.0007$ ). Approximately $30 \%$ of respondents reported a history of uveitis, and 57 respondents with AS indicated that they had at least a history of psoriasis.

On average, participants first noticed disease symptoms 26.6 years prior to the completion of the survey and received an AS diagnosis $\approx 8.2$ years later. AS symptoms most commonly emerged when participants were aged $16-20$ years $(21.6 \%)$, and diagnoses were most commonly made when participants were 26-30 years of age $(16.7 \%)$. Of the 865 participants who completed the 2011 LIS, 788 (91.1\%) selfreported receiving a diagnosis of AS; the baseline demographics and disease characteristics of these respondents (weighted) are shown in Table S1 of the Electronic supplementary material (ESM).

On average, respondents with AS had consulted with 2.2 doctors about their back pain, joint pain, or inflammatory problems within the last 2 years; this included $20.7 \%$ of respondents who had seen $\geq 4$ doctors in that period. With regard to current treatment, $76.7 \%$ of respondents with AS received care from rheumatologists, $8.7 \%$ from primary care physicians, and $3.8 \%$ from orthopedic surgeons (Table 2). More than one-third $(36.7 \%)$ of respondents saw their doctor 2-3 times a year, and $33.0 \%$ saw their doctor every $2-3$ months; $57.5 \%$ of respondents discussed medication options with their doctor and jointly participated in treatment decisions. Of the 52 respondents $(7.3 \%)$ who were not currently seeing a doctor for their AS, 17 reported that their disease did not bother them enough to obtain medical help.

Respondents with AS were most commonly currently receiving biologics $(50.7 \%)$, NSAIDs $(31.6 \%)$, analgesics $(23.3 \%)$, and slow-acting antirheumatic drugs $(18.2 \%)$ at the time of the survey (Table 2). In general, women reported more drug use than men in all 4 categories ( $54.7 \%$ vs $46.1 \%, 36.6 \%$ vs $25.9 \%, 28.4 \%$ vs $17.6 \%$, and $24.2 \%$ vs $11.3 \%$, respectively). Of 364 respondents with AS who were queried about the primary reason for choosing their specific biologic, $45.3 \%$ reported that they were using it because it was recommended by their doctor. Overall, most respondents (63.7\%) were satisfied ("slightly," "somewhat," "mostly," and "completely satisfied") with their current drug treatment. Many respondents also applied nonmedicinal treatment to their lifestyle, including stretching or strengthening exercises (66.1\%); biking, running, or walking $(58.1 \%)$; practicing proper posture techniques $(55.3 \%)$; and special diets $(39.0 \%)$. A summary of medical care, treatment history, and satisfaction with treatment taken at the time of survey participation in the 2011 LIS (weighted) is shown in Table 52 of the ESM.

Using the EASi-QoL survey, respondents reported the impact of AS on their QOL (Fig. 2). The mean total EASi-QoL score was 28.9 
Table 2 Summary of medical care, current treatment, and satisfaction with current treatment of respondents with AS in the 2017 Life Impact Survey

\begin{tabular}{ll}
\hline Characteristic & $\begin{array}{l}\text { Respondents with } \\
\text { AS, } N=716\end{array}$ \\
\hline $\begin{array}{l}\text { Type of doctor seen for treatment, } \\
n \text { (\%) }\end{array}$ & $N=716$ \\
Rheumatologist & $549(76.7)$ \\
Primary care physician & $62(8.7)$ \\
Orthopedic surgeon & $27(3.8)$ \\
Physical therapist & $8(1.1)$ \\
Chiropractor & $9(1.3)$ \\
Gastroenterologist & $6(0.8)$ \\
Ophthalmologist & $2(0.3)$ \\
Obstetrician/gynecologist & $1(0.1)$ \\
Not in treatment & $52(7.3)$ \\
Frequency of doctor visits, $n(\%)$ & $N=716$ \\
Weekly or more often & $2(0.3)$ \\
Several times a month & $11(1.5)$ \\
Once a month & $43(6.0)$ \\
Every 2-3 months & $236(33.0)$ \\
2-3 times a year & $263(36.7)$ \\
Once a year & $74(10.3)$ \\
Less often than yearly & $70(9.8)$ \\
Not sure & $17(2.4)$ \\
Reasons for not seeing a doctor for & $N=52$ \\
AS treatment, $n$ (\%) & \\
My disease does not bother me & $17(32.7)$ \\
enough & \\
In remission/controlled/passed & $10(19.2)$ \\
away & \\
Given up & $(19.2)$ \\
Cost & \\
effects of medication & \\
\hline
\end{tabular}

Table 2 continued

\begin{tabular}{|c|c|}
\hline Characteristic & $\begin{array}{l}\text { Respondents with } \\
\text { AS, } N=716\end{array}$ \\
\hline Too much hassle & $5(9.6)$ \\
\hline $\begin{array}{l}\text { No one in area who is experienced } \\
\text { in treating AS }\end{array}$ & $4(7.7)$ \\
\hline Too far away & $2(3.8)$ \\
\hline Other & $7(13.5)$ \\
\hline Current drug treatment, $n(\%)$ & $N=716$ \\
\hline Analgesics (acetaminophen) & $167(23.3)$ \\
\hline Biologics & $363(50.7)$ \\
\hline Adalimumab & $125(17.5)$ \\
\hline Certolizumab pegol & $25(3.5)$ \\
\hline Etanercept & $72(10.1)$ \\
\hline Golimumab & $16(2.2)$ \\
\hline Infliximab & $82(11.5)$ \\
\hline Secukinumab & $45(6.3)$ \\
\hline Cannabis (medical marijuana) & $50(7.0)$ \\
\hline NSAIDs & $226(31.6)$ \\
\hline Ibuprofen & $138(19.3)$ \\
\hline Indomethacin & $28(3.9)$ \\
\hline Naproxen & $90(12.6)$ \\
\hline Opioids & $99(13.8)$ \\
\hline Hydrocodone & $58(8.1)$ \\
\hline Oxycodone & $51(7.1)$ \\
\hline SAARDs & $130(18.2)$ \\
\hline Methotrexate & $78(10.9)$ \\
\hline Sulfasalazine & $67(9.4)$ \\
\hline Steroids & $96(13.4)$ \\
\hline Cortisone & $20(2.8)$ \\
\hline Hydrocortisone & $22(3.1)$ \\
\hline Prednisone & $71(9.9)$ \\
\hline Other & $255(35.6)$ \\
\hline
\end{tabular}


Table 2 continued

\begin{tabular}{|c|c|}
\hline Characteristic & $\begin{array}{l}\text { Respondents with } \\
\text { AS, } N=716\end{array}$ \\
\hline $\begin{array}{l}\text { Overall satisfaction with current } \\
\text { drug treatment, } n(\%)\end{array}$ & $N=716$ \\
\hline Not satisfied & $51(7.1)$ \\
\hline Slightly satisfied & $64(8.9)$ \\
\hline Somewhat satisfied & $150(20.9)$ \\
\hline Mostly satisfied & $280(39.1)$ \\
\hline Completely satisfied & $112(15.6)$ \\
\hline Unsure/not available & $59(8.2)$ \\
\hline $\begin{array}{l}\text { Current nonmedicinal treatment } \\
\text { for AS, } n(\%)\end{array}$ & $N=716$ \\
\hline $\begin{array}{l}\text { Stretching and strengthening } \\
\text { exercises }\end{array}$ & $473(66.1)$ \\
\hline Biking/running/walking & $416(58.1)$ \\
\hline Proper posture techniques & $396(55.3)$ \\
\hline Special diet & $279(39.0)$ \\
\hline Breathing exercises & $252(35.2)$ \\
\hline Quit smoking & $199(27.8)$ \\
\hline Swimming/water exercises & $175(24.4)$ \\
\hline Sports & $138(19.3)$ \\
\hline Yoga & $113(15.8)$ \\
\hline Dance & $55(7.7)$ \\
\hline Tai chi & $23(3.2)$ \\
\hline Other & $57(8.0)$ \\
\hline
\end{tabular}

$A S$ ankylosing spondylitis, NSAIDs nonsteroidal anti-inflammatory drugs, $S A A R D$ s slow-acting antirheumatic drugs

(weighted); overall, on the basis of weighted data, $33.7 \%, 31.7 \%$, and $34.7 \%$ of respondents, respectively, reported a low (EASi-QoL score $0-17)$, medium (18-35), and high ( $\geq 36$ ) impact of AS on QOL. These numbers are comparable to those of the 2011 LIS; the mean total EASiQoL score in the 2011 LIS was 26.9 (weighted), and $37.4 \%, 31.3 \%$, and $31.3 \%$ of respondents, respectively, reported a low, a medium, and a high impact of AS on QOL (Fig. S1 in the ESM). Of the four QOL domains evaluated, AS had the highest impact on physical function in both LISs, with $41.9 \%$ and $37.0 \%$ of respondents overall (weighted) reporting a high impact of AS on the physical domain (EASi-QoL score $\geq 10$ ) in 2017 and 2011, respectively. When respondents with AS were stratified by sex, women were more likely than men to report a high impact of disease across all EASi-QoL questions (45.1\% vs $31.2 \% ; P=0.0006)$. This observation extended to individual domains, for which women were also significantly more likely than men to report a high impact of disease on the physical $(50.3 \%$ vs $39.2 \% ; P=0.0098)$, discomfort $(39.0 \%$ vs $26.8 \% ; P=0.0017)$, emotional (35.2\% vs $24.2 \% ; P=0.0023)$, and social (40.5\% vs $29.9 \% ; P=0.0031$ ) domains (Fig. $2 \mathrm{a}$ ). Similar trends were observed in the 2011 LIS for respondents stratified by sex (Fig. $\mathrm{S} 1$ in the ESM). When stratified by NSAID vs biologic use, a lower proportion of respondents on biologics reported a high impact of AS on their QOL (Fig. 2b).

The proportion of respondents (overall and stratified by sex) reporting a high impact of AS (i.e., the top two levels of impairment for each question) in the four QOL domains is shown in Fig. 3. Women were more likely than men to report a high impact of AS in several aspects in all QOL domains. In the physical domain, significantly more women than men reported being negatively impacted by AS with regard to lifting a child or heavy objects $(43.2 \%$ vs $27.8 \%$; $P<0.0001)$ and standing for $30 \mathrm{~min}(34.9 \%$ vs $26.0 \% ; P=0.0128)$. In the discomfort domain, significantly more women than men reported feeling tired or lacking in energy $(46.4 \%$ vs $33.4 \% ; P=0.0004)$ and interference with sleep $(32.7 \%$ vs $23.4 \% ; P=0.0081)$. In the emotional domain, significantly more women than men were worried about the future $(37.3 \%$ vs $23.5 \%$; $P<0.0001)$, lacked drive or motivation $(27.2 \%$ vs $20.4 \% ; P=0.0364$ ), and found that AS interfered with their ability to concentrate (19.8\% vs $12.0 \% ; P=0.0057)$. Last, in the social domain, significantly more women than men reported that their AS prevented them from keeping physically active $(35.9 \%$ vs $26.6 \% ; P$ $=0.0092$ ), prevented them from traveling by car 


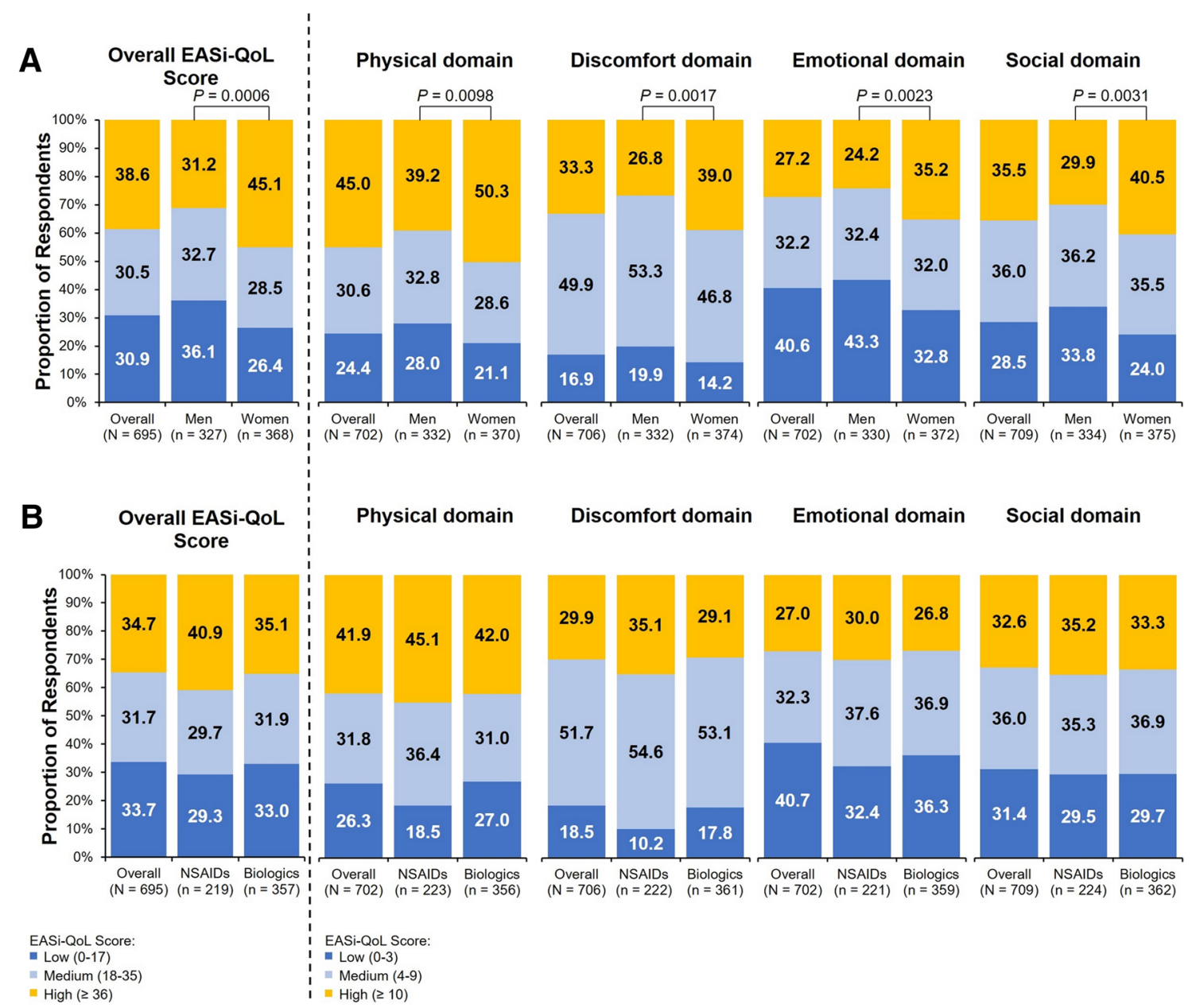

Fig. 2 Impact of AS on the QOL of respondents with AS stratified by a sex and $\mathbf{b}$ NSAID vs biologic use are reported as observed. Data stratified by current NSAID vs biologic use are weighted 3:1 (male to female). $P<0.05$ is considered statistically significant. $A S$ ankylosing

or public transport $(19.2 \%$ vs $10.5 \%$; $P$ $=0.0019)$, and interfered with their normal work $(30.7 \%$ vs $23.1 \% ; P=0.0268)$. Similar trends were observed among respondents in the 2011 LIS (Fig. S2 in the ESM).

The impact of AS on certain lifestyle characteristics is shown in Fig. 4, including participation in sports, career choice, ability to work, and time with friends and family. Nearly twothirds $(65.1 \%)$ of respondents reported a frequent ("all the time" and "often") impact of AS on participation in sports. In addition, $\approx 40 \%$ of respondents reported similar impacts of AS on career choice and how much they were able spondylitis; EASi-QOL Evaluation of Ankylosing Spondylitis Quality of Life questionnaire; NSAID nonsteroidal anti-inflammatory drug; $Q O L$ quality of life. ${ }^{*} P<0.05$, male vs female

to work, and nearly one-third (31.3\%) reported that AS impacted the time spent with friends and family.

\section{DISCUSSION}

In this updated 2017 LIS of 716 respondents with AS from the SAA, we found that AS imposes negative impacts in all QOL domains analyzed (physical, discomfort, emotional, and social); the mean overall weighted EASi-QoL score was 28.9. Furthermore, women with AS were significantly more impacted than men in 


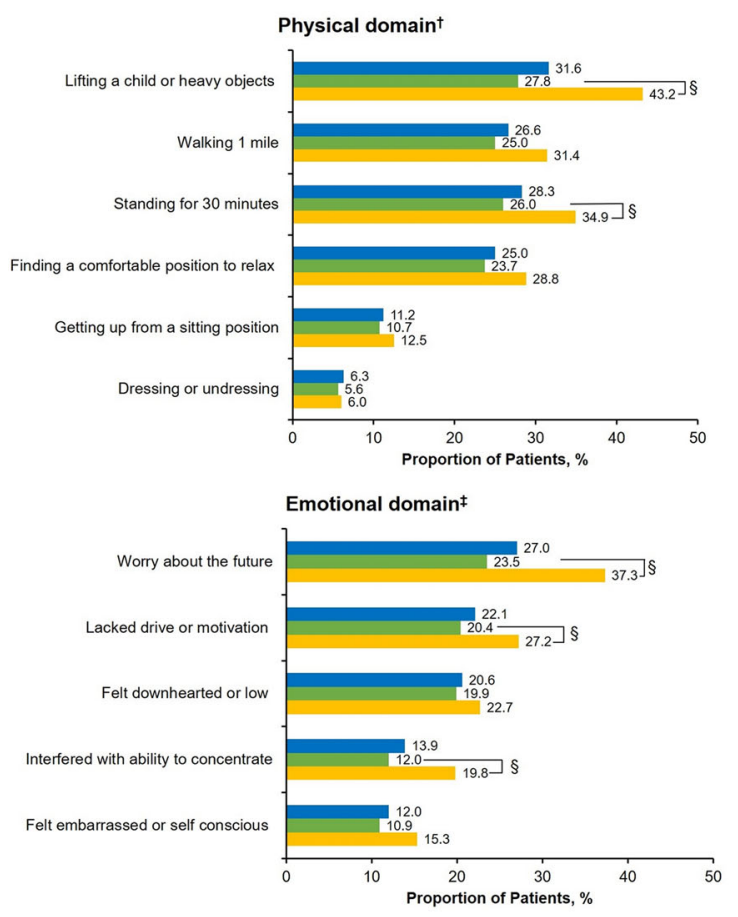

Fig. 3 Proportion of respondents with AS reporting the top two levels of impairment by their disease in activities across four domains of disease stratified by sex in the 2017 Life Impact Survey. Respondents were "very limited" or "totally limited/unable to do" each activity as a result of their AS. $P<0.05$ is considered statistically significant. $A S$ ankylosing spondylitis; $Q O L$ quality of life. ${ }^{*}$ Weighted 3:1

several aspects across all QOL domains. Overall, AS had the highest negative impact on physical function and affected various lifestyle characteristics, including participation in sports and career choice.

Our findings from 2017 share many similarities with the results of the 2011 LIS. In 2011, the mean overall weighted EASi-QoL score was 26.9 among all respondents with AS, and respondents reported negative impacts of AS in all QOL domains. Interestingly, the mean overall weighted EASi-QoL score was higher in 2017 than in 2011 (28.9 vs 26.9), as was the proportion of respondents who reported a high impact of disease on QOL (34.7\% vs 31.3\%). Respondents were older and shared a higher comorbidity burden in 2017 vs 2011, which may have contributed to the slightly higher impact of disease on QOL. With regard to comorbidities and AS-associated diseases, the

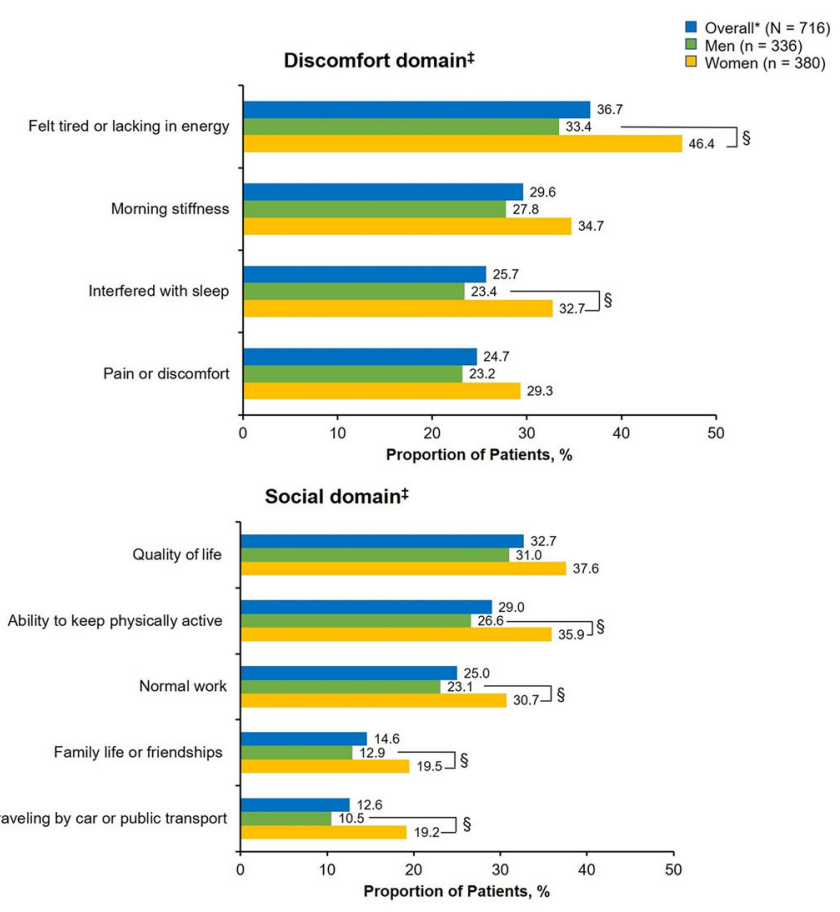

(male to female). ${ }^{\dagger}$ For activities related to the physical domain, participants were queried about the impact of AS on their QOL on the day of survey participation. ${ }^{\star}$ For activities related to the discomfort, emotional, and social domains, participants were queried about the impact of AS on their QOL during the previous week. ${ }^{\S} P<0.05$, male vs female

proportions of respondents reporting high blood pressure and high cholesterol are similar in both surveys. However, uveitis was more frequently reported by respondents in 2017 vs 2011 (30.3\% vs $19.0 \%$ ), and eye inflammation was more commonly reported than uveitis in both surveys (46.4\% in 2017 and $47.9 \%$ in 2011). "Eye inflammation" is a broad term that includes uveitis [31], and few may recognize the term "uveitis." It is therefore possible that respondents reporting eye inflammation may have experienced uveitis but reported eye inflammation due to unfamiliarity with the term "uveitis." As similarly observed in the 2017 LIS, more women than men reported a high impact of AS in all four domains in the 2011 LIS across all individual activities except getting up from a sitting position, dressing or undressing, and feeling embarrassed or self-conscious; a significantly higher proportion of women than 


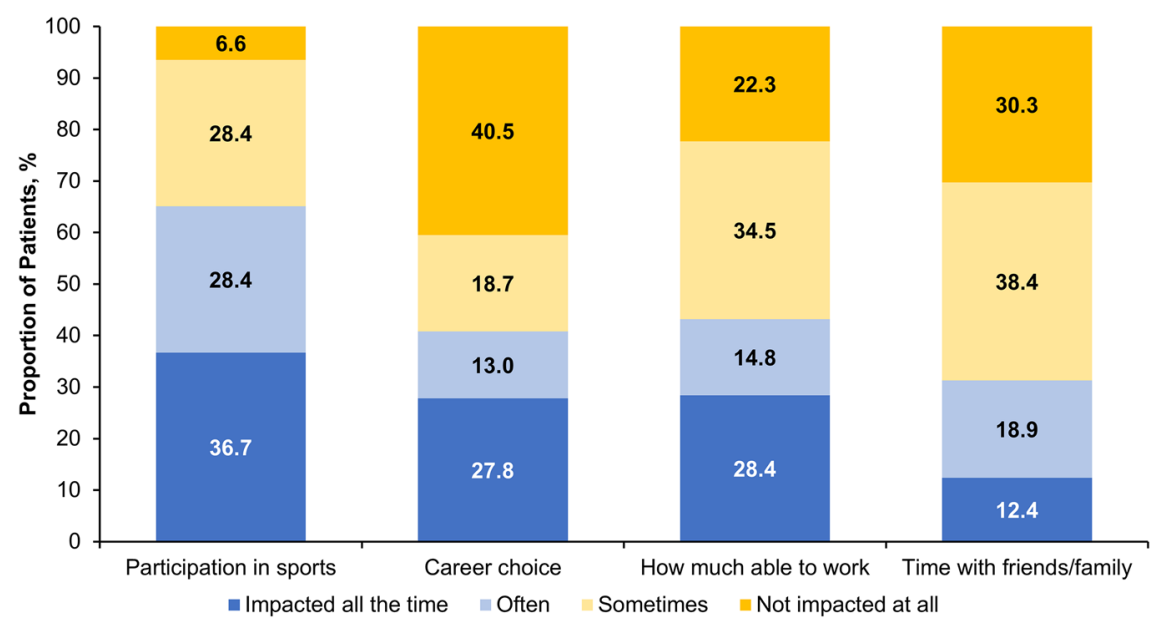

Fig. 4 Patient-reported impact of AS on lifestyle characteristics in the 2017 Life Impact Survey $(N=716)$. Data are weighted 3:1 (male:female). $A S$ ankylosing spondylitis

men with AS reported difficulties standing for $30 \mathrm{~min}$. With regard to medication, respondents in 2017 had more treatment options available compared with respondents in 2011, particularly newer biologics. Most respondents in both surveys reported being under the care of rheumatologists and using other nonmedicinal treatments such as strengthening exercises and yoga.

Our results mirror those of a study reviewing the QOL of patients with SpA-European patients with AS also reported that the disease had the most impact on physical domains [32]. Spinal pain and mobility restriction, which may be measured by the Bath Ankylosing Spondylitis Functional Index (BASFI), inflict substantial impairments on daily activities [33]; BASFI metrics correlated substantially with QOL measurements $[32,34]$. The severity and symptoms of AS, along with pain, discomfort, and fatigue, were the primary contributors to impairment of QOL [34]. Overall, approximately one-third of survey respondents reported feeling tired or lacking in energy, more significantly among women than men. Indeed, the management of pain and fatigue are crucial to alleviating the physical, social, and psychological aspects of AS [35].

The advent of biologic agents has revolutionized the AS treatment landscape, and current evidence indicates that routine clinical use of biologics greatly improves patient QOL [10]. Since biologics are more likely to be prescribed for patients with more severe disease $[10,36]$, and respondents on biologics in our study had QOL scores roughly comparable to those on NSAIDs, our data imply that biologics resulted in a substantial improvement in QOL. However, the clinical benefits of biologics may be diminished due to the poor treatment adherence and early discontinuation, thus increasing medical costs and forcing the need for more aggressive treatments [37].

Depression and anxiety are also frequently reported among patients with AS [34, 38]; in fact, even though emotional issues were the second most commonly discussed topic among patients with AS in online communities, the ability of commonly used patient-reported outcome tools to capture these issues was limited [38]. In our study, anxiety about the future was the most frequently reported impairment in the emotional domain (27.0\% overall), more significantly among women $(37.3 \%)$ than men $(23.5 \% ; P<0.05)$. A decade ago, screening for depression among patient populations was discouraged unless clinicians could establish that it would improve health outcomes [39]; now, with the realization that chronic pain-related diseases are often linked to mental health disorders [40], an integrated, multidisciplinary 
approach to treatment inclusive of psychiatric support should be recommended.

The ability to keep physically active $(29.0 \%)$ and interference with work $(25.0 \%)$ and family life or friendships (14.6\%) are some of the aspects of the social domain reported by survey respondents to be impacted by AS. These aspects, along with traveling by car or public transportation $(12.6 \%$ overall), were significantly more impacted in women $(19.2 \%)$ than in men $(10.5 \% ; P<0.05)$. Our results agree with those of a cross-sectional survey regarding work and family life, which reported that patients with AS in several US metropolitan cities were significantly more likely than the general population to be work disabled, unemployed, and divorced or never married; women with AS were less likely to have children, but the number of men with AS who had children was not different from that of the general population [41]. Likewise, a more recent study reported that patients with AS were more frequently dissatisfied and extremely dissatisfied with life compared with controls, and highlighted the importance of providing support with regard to social participation in order to improve QOL [42].

Significant sex differences were observed with regard to comorbid conditions; e.g., women were more likely than men to have comorbid depression and fibromyalgia, while men were more likely than women to have comorbid hypertension and heart disease. These comorbidities could very likely play a role in sex differences in QOL. Additionally, sex differences observed across all QOL domains examined in our analysis have been discussed in several other studies, highlighting that female patients may manifest AS differently from men due to different immunologic [43] and genetic $[44,45]$ responses to the disease. Furthermore, disease activity as measured by the Bath Ankylosing Spondylitis Disease Activity Index was significantly higher in female than in male patients with AS in several studies [46-48]. One report also documented a significantly lower QOL in female than in male patients as measured by the Ankylosing Spondylitis Quality of Life questionnaire [47].
As with all survey-based studies, patient perspectives may be subject to the patients' bias and experience. Physician-confirmed diagnoses and detailed treatment histories were not available; our study relied on respondents' selfreported physician-made diagnoses of AS and other SpA conditions, which may lead to underor over-representation of associated conditions and/or bias in reporting of symptoms. It is unlikely that the absence of physician-confirmed diagnoses invalidates the study, as past studies have shown that patients are reasonably accurate in their self-diagnosis of SpA $[49,50]$; the symptoms and medication use reported by survey respondents strongly indicate spondylitis. The survey participants may be more motivated and/or involved in their healthcare than patients with AS in the general US population. While the overall participation rate was high (our study was planned for 800 respondents700 by online survey and 100 by phone), the participation rate was greater among female than among male respondents, which is consistent with other studies showing that women are more likely than men to participate in online studies [38, 51].

\section{CONCLUSIONS}

This real-world survey targeted patients with AS across the United States, thus providing greater representation of the geographic distribution, demographic diversity, and disease spectrum compared with tertiary referral centers or clinical trials. Respondents with AS reported negative impacts in all QOL domains, with a mean overall EASi-QoL score of 28.9. Sex differences showing a greater impact of AS on QOL in women versus men were noted across several aspects of daily living, including lifting a child or heavy objects and worrying about the future. Integrating subjective metrics of disease via patient-reported outcomes should be included, along with clinical evaluation of AS progression. Overall, available studies, including ours, point to the need to identify and ameliorate impaired QOL aspects to improve the outcomes of disabling rheumatological disorders such as AS. 


\section{ACKNOWLEDGEMENTS}

Funding. This study was conducted by the Spondylitis Association of America and funded by Novartis Pharmaceuticals Corporation and $\mathrm{UCB}$, Inc. The article processing charges were also funded by Novartis.

Medical Writing and Editorial Assistance. Support for third-party writing assistance for this manuscript, furnished by Kheng Bekdache, PhD, of Health Interactions, Inc., was provided by the Spondylitis Association of America.

Authorship. All named authors meet the International Committee of Medical Journal Editors (ICMJE) criteria for authorship for this article, take responsibility for the integrity of the work as a whole, and have given their approval for this version to be published. All authors had full access to all of the data in this study and take complete responsibility for the integrity of the data and accuracy of the data analysis.

Disclosures. J. Rosenbaum receives research support from Alcon Research Institute, the Spondylitis Association of America (SAA), and Pfizer; consults for AbbVie, Eyevensys, Gilead, Janssen, Novartis, Regeneron, Roche, and UCB; and receives royalties from UpToDate. L. Pisenti is an employee of UCB, Inc. Y. Park is an employee of Novartis Pharmaceuticals Corporation. R. Howard is an employee of the SAA.

Compliance with Ethics Guidelines. IRB approval was not obtained when this study was designed in 2011. The IRB of Oregon Health \& Science University has since confirmed that the study design was compliant with the Declaration of Helsinki and the Belmont Report.

Data Availability. The datasets generated during and/or analyzed during the current study are proprietary and not publicly available; however, requests for datasets should be sent to the Spondylitis Association of America.
Open Access. This article is distributed under the terms of the Creative Commons Attribution-NonCommercial 4.0 International License (http://creativecommons.org/licenses/ by-nc/4.0/), which permits any noncommercial use, distribution, and reproduction in any medium, provided you give appropriate credit to the original author(s) and the source, provide a link to the Creative Commons license, and indicate if changes were made.

\section{REFERENCES}

1. Rudwaleit M, Landewe $R$, van der Heijde $D$, et al. The development of Assessment of SpondyloArthritis international Society classification criteria for axial spondyloarthritis (part I): classification of paper patients by expert opinion including uncertainty appraisal. Ann Rheum Dis. 2009;68: 770-6.

2. Reveille JD, Weisman MH. The epidemiology of back pain, axial spondyloarthritis and HLA-B27 in the United States. Am J Med Sci. 2013;345:431-6.

3. Dougados M, Baeten D. Spondyloarthritis. Lancet. 2011;377:2127-37.

4. Kang JH, Chen YH, Lin HC. Comorbidity profiles among patients with ankylosing spondylitis: a nationwide population-based study. Ann Rheum Dis. 2010;69:1165-8.

5. Oldroyd J, Schachna L, Buchbinder R, et al. Ankylosing spondylitis patients commencing biologic therapy have high baseline levels of comorbidity: a report from the Australian Rheumatology Association database. Int J Rheumatol. 2009;2009:268569.

6. Bremander A, Petersson IF, Bergman S, Englund M. Population-based estimates of common comorbidities and cardiovascular disease in ankylosing spondylitis. Arthritis Care Res (Hoboken). 2011;63:550-6.

7. Shen CC, Hu LY, Yang AC, Kuo BI, Chiang YY, Tsai SJ. Risk of psychiatric disorders following ankylosing spondylitis: a nationwide population-based retrospective cohort study. J Rheumatol. 2016;43: 625-31.

8. Rosenbaum JT. Uveitis in spondyloarthritis including psoriatic arthritis, ankylosing spondylitis, and inflammatory bowel disease. Clin Rheumatol. 2015;34:999-1002. 
9. Ward MM, Deodhar A, Akl EA, et al. American College of Rheumatology/Spondylitis Association of America/Spondyloarthritis Research and Treatment Network 2015 recommendations for the treatment of ankylosing spondylitis and nonradiographic axial spondyloarthritis. Arthritis Rheumatol. 2016;68:282-98.

10. van der Heijde D, Ramiro S, Landewe R, et al. 2016 update of the ASAS-EULAR management recommendations for axial spondyloarthritis. Ann Rheum Dis. 2017;76:978-91.

11. Maruotti N, d'Onofrio F, Cantatore FP. Metabolic syndrome and chronic arthritis: effects of anti-TNF$\alpha$ therapy. Clin Exp Med. 2015;15:433-8.

12. van Tubergen A, Weber U. Diagnosis and classification in spondyloarthritis: identifying a chameleon. Nat Rev Rheumatol. 2012;8:253-61.

13. Eilertsen G, Ormstad H, Kirkevold M, Mengshoel AM, Söderberg S, Olsson M. Similarities and differences in the experience of fatigue among people living with fibromyalgia, multiple sclerosis, ankylosing spondylitis and stroke. J Clin Nurs. 2015;24:2023-34.

14. Ojala T, Häkkinen A, Karppinen J, Sipilä K, Suutama $\mathrm{T}$, Piirainen A. Chronic pain affects the whole person-a phenomenological study. Disabil Rehabil. 2015;37:363-71.

15. Dow CM, Roche PA, Ziebland S. Talk of frustration in the narratives of people with chronic pain. Chronic Ill. 2012;8:176-91.

16. Deodhar A, Mittal M, Reilly P, et al. Ankylosing spondylitis diagnosis in US patients with back pain: identifying providers involved and factors associated with rheumatology referral delay. Clin Rheumatol. 2016;35:1769-76.

17. Sieper J, Rudwaleit M. Early referral recommendations for ankylosing spondylitis (including pre-radiographic and radiographic forms) in primary care. Ann Rheum Dis. 2005;64:659-63.

18. Braun J, Sieper J. Early diagnosis of spondyloarthritis. Nat Clin Pract Rheumatol. 2006;2: 536-45.

19. Boonen A, van der Linden SM. The burden of ankylosing spondylitis. J Rheumatol Suppl. 2006; 78:4-11.

20. González CM, Carmona L, de Toro J, et al. Perceptions of patients with rheumatic diseases on the impact on daily life and satisfaction with their medications: RHEU-LIFE, a survey to patients treated with subcutaneous biological products. Patient Prefer Adherence. 2017;11:1243-52.
21. Ware JE Jr, Sherbourne CD. The MOS 36-item shortform health survey (SF-36). I. Conceptual framework and item selection. Med Care. 1992;30: 473-83.

22. Doward LC, Spoorenberg A, Cook SA, et al. Development of the ASQoL: a quality of life instrument specific to ankylosing spondylitis. Ann Rheum Dis. 2003;62:20-6.

23. Haywood KL, Garratt AM, Jordan KP, Healey EL, Packham JC. Evaluation of ankylosing spondylitis quality of life (EASi-QoL): reliability and validity of a new patient-reported outcome measure. J Rheumatol. 2010;37:2100-9.

24. Baysal O, Durmuş B, Ersoy Y, et al. Relationship between psychological status and disease activity and quality of life in ankylosing spondylitis. Rheumatol Int. 2011;31:795-800.

25. Bodur H, Ataman S, Rezvani A, et al. Quality of life and related variables in patients with ankylosing spondylitis. Qual Life Res. 2011;20:543-954.

26. Ward MM. Health-related quality of life in ankylosing spondylitis: a survey of 175 patients. Arthritis Care Res. 1994;12:247-55.

27. Davis JCJ, Mease PJ. Insights into the pathology and treatment of spondyloarthritis: from the bench to the clinic. Semin Arthritis Rheum. 2008;38:83-100.

28. Stone M, Warren RW, Bruckel J, Cooper D, Cortinovis D, Inman RD. Juvenile-onset ankylosing spondylitis is associated with worse functional outcomes than adult-onset ankylosing spondylitis. Arthritis Rheum. 2005;53:445-51.

29. Spondylitis Association of America. http://www. saa.org. Accessed 13 Feb 2019.

30. Reveille JD, Witter JP, Weisman MH. Prevalence of axial spondylarthritis in the United States: estimates from a cross-sectional survey. Arthritis Care Res (Hoboken). 2012;64:905-10.

31. Thorne DE, Jabs DA. Rheumatic diseases. In: Ryan SJ, editor. The retina. 4th ed. Oxford: Elsevier; 2006. p. $1383-408$.

32. Torre-Alonso JC, Queiro R, Comellas M, Lizán L, Blanch C. Patient-reported outcomes in European spondyloarthritis patients: a systematic review of the literature. Patient Prefer Adher. 2018;12: 733-47.

33. van Echteld I, Cieza A, Boonen A, et al. Identification of the most common problems by patients with ankylosing spondylitis using the international classification of functioning, disability and health. J Rheumatol. 2006;33:2475-83. 
34. Kotsis K, Voulgari PV, Drosos AA, Carvalho AF, Hyphantis T. Health-related quality of life in patients with ankylosing spondylitis: a comprehensive review. Expert Rev Pharmacoecon Outcomes Res. 2014;14:857-72.

35. Brophy S, Davies H, Dennis MS, et al. Fatigue in ankylosing spondylitis: treatment should focus on pain management. Semin Arthritis Rheum. 2013;42:361-7.

36. Sepriano A, Regel A, van der Heijde D et al (2017) Efficacy and safety of biological and targeted-synthetic DMARDs: a systematic literature review informing the 2016 update of the ASAS/EULAR recommendations for the management of axial spondyloarthritis. RMD Open. 2017;3(1):e000396.

37. Walsh JA, Adejoro O, Chastek B, Park Y. Treatment patterns of biologics in US patients with ankylosing spondylitis: descriptive analyses from a claims database. J Comp Eff Res. 2018;7:369-80.

38. Sunkureddi P, Gibson D, Doogan S, Heid J, Benosman S, Park Y. Using self-reported patient experiences to understand patient burden: learnings from digital patient communities in ankylosing spondylitis. Adv Ther. 2018;35:424-37.

39. Gilbody S, Sheldon T, House A. Screening and casefinding instruments for depression: a meta-analysis. CMAJ. 2008;178:997-1003.

40. Esen SA, Esen I, Karabulut Y, Atmiş V. Effects of the disease characteristics and the treatment on psychological status in patients with rheumatoid arthritis and ankylosing spondylitis. Curr Rheumatol Rev. 2017;14:271-8.

41. Ward MM, Reveille JD, Learch TJ, Davis JC Jr, Weisman MH. Impact of ankylosing spondylitis on work and family life: comparisons with the US population. Arthritis Rheum. 2008;59:497-503.

42. van Genderen S, Plasqui G, van der Heijde D, et al. Social role participation and satisfaction with life: a study among patients with ankylosing spondylitis and population controls. Arthritis Care Res (Hoboken). 2018;70:600-7.
43. Gracey E, Yao Y, Green B, et al. Sexual dimorphism in the Th17 signature of ankylosing spondylitis. Arthritis Rheumatol. 2016;68:679-83.

44. Tsui HW, Inman RD, Paterson AD, Reveille JD, Tsui FW. ANKH variants associated with ankylosing spondylitis: gender differences. Arthritis Res Ther. 2005;7:R513-25.

45. Tsui HW, Inman RD, Reveille JD, Tsui FW. Association of a TNAP haplotype with ankylosing spondylitis. Arthritis Rheum. 2007;56:234-43.

46. van der Horst-Bruinsma IE, Zack DJ, Szumski A, Koenig AS. Female patients with ankylosing spondylitis: analysis of the impact of gender across treatment studies. Ann Rheum Dis. 2013;72: 1221-4.

47. Webers C, Essers I, Ramiro S, et al. Gender-attributable differences in outcome of ankylosing spondylitis: long-term results from the Outcome in Ankylosing Spondylitis International Study. Rheumatology (Oxford). 2016;55:419-28.

48. Glintborg B, Ostergaard M, Krogh NS, Dreyer L, Kristensen HL, Hetland ML. Predictors of treatment response and drug continuation in 842 patients with ankylosing spondylitis treated with anti-tumour necrosis factor: results from 8 years' surveillance in the Danish nationwide DANBIO registry. Ann Rheum Dis. 2010;69:2002-8.

49. Barlow JH, Turner AP, Wright CC. Comparison of clinical and self-reported diagnoses for participants on a community-based arthritis self-management programme. Br J Rheumatol. 1998;37:985-7.

50. Saraux A, Guillemin F, Fardellone P, et al. Agreement between rheumatologist visit and lay interviewer telephone survey for screening for rheumatoid arthritis and spondyloarthropathy. Joint Bone Spine. 2004;71:44-50.

51. PatientsLikeMe: Ankylosing Spondylitis. https:// www.patientslikeme.com/conditions/201-ankylosi ng-spondylitis. Accessed 13 Feb 2019. 